\title{
On Dipole Moments and Hydrogen Bond Identification in Water Clusters
}

\author{
Imre BAKÓ* and István MAYER \\ Research Centre for Natural Sciences, Hungarian Academy of Sciences, \\ H-1519 Budapest, P.O.Box 286, Hungary
}

\begin{abstract}
It is demonstrated that the localized orbitals calculated for a water cluster have small delocalization tails along the hydrogen bonds, that are crucial in determining the resulting dipole moments of the system. (By cutting them, one gets much smaller dipole moments for the individual monomers - close to the values one obtains by using a Badertype analysis.) This means that the individual water monomers can be delimited only in a quite fuzzy manner, and the electronic charge density in a given point cannot be assigned completely to that or another molecule. Thus one arrives to the brink of breaking the concept of a water cluster consisting of individual molecules. The analysis of the tails of the localized orbitals can also be used to identify the pairs of water molecules actually forming hydrogen bonds.
\end{abstract}

*E-mail: bako.imre@ttk.mta.hu; phone (+361) 382-6981 


\section{Introduction}

It is customary to consider liquid (or solid) water as a conglomerate of distinct water molecules connected with a network of hydrogen bonds. Each individual water molecule is assumed to carry a considerable dipole moment, which is significantly larger than that of a free water molecule due to collective effects: the experimental dipole moment ${ }^{1}$ of a water molecule in water medium $^{2-4}$ is about 2.8-2.95 Debye (D), as compared with the dipole moment of ca. $1.85 \mathrm{D}$ of a free water monomer ${ }^{5}$. The increase of the dipole moment takes place already for small water clusters: the authors of Ref. 6. measured by using terahertz laser vibration-rotation-tunneling spectroscopy the dipole moment of the cage structure of the water hexamer cluster and performed ab initio and iterated induction model calculations for small (up to 8 water molecules) gas phase clusters. They concluded that the average dipole moment is increased as a function of number of water molecules in the cluster. The dipole moment of water monomer in cage like structure of cyclic hexamer was found to be about 2.70 D. That result indicates that this collective effect can be well studied by considering relatively small water clusters; this facilitates obtaining a deeper insight into its nature by performing different calculations on model water clusters.

One may be interested in determining the actual value of the dipole moments of different individual water molecules in different water models. Then one has to take into account two requirements the fulfillment of which is highly desirable. First, dipole moment should be calculated for a neutral entity, otherwise - as we know from electrostatics - the dipole moment becomes ill-defined (depends on the selection of the origin of the coordinate system). Second: the dipole moments of the constituents should sum to the overall dipole moment of the cluster. As will be seen further, these requirements put one on the brink of breaking the concept of a water cluster consisting of individual molecules.

There are three approaches in the literature for calculating dipole moments of a monomer within a water model. In the simplest one considers a single water molecule described quantum mechanically, which is embedded into an environment of point charges and/or multipoles determined by different assumptions. In some cases this embedded system is considered also solvated. These schemes ${ }^{7-13}$ give $2.6-2.8 \mathrm{D}$ for the dipole of a water monomer, depending on the details of calculations. Even this scheme can account for the significant increase of the dipole moment as compared with a single monomer.

The other two schemes utilize the electron density calculated from the global wave function of a cluster, either of finite size or in a periodic descrip- 
tion. One of them can be accomplished at any level of theory, and requires performing a 3-dimensional (3D) "topological" Bader analysis ${ }^{14,15}$, with the help of which the 3D space is decomposed into atomic domains by studying the charge distribution, and a domain of an oxygen atom is combined with those of the respective two hydrogen atoms to form a domain of a water monomer ${ }^{16}$. Then the dipole moment of a water molecule is obtained by integrating the charge density in the domain in the molecule in question, taking the oxygen nucleus as the origin. Although this approach does not strictly fulfill the first requirement, that is assumed not to cause a big error, as the oxygen atom is close to the center of the charge of a water molecule.

Another scheme was applied by some authors in the framework of plane wave calculations of water clusters: its essence is in turning to a Wannierfunction basis representing linear combinations of plane waves, assigning the different Wannier functions to the individual monomers and calculating dipole moments by their use ${ }^{17-20}$. This scheme fulfills, at less formally, both requirements mentioned above and, as a rule of thumb, results in some $0.5 \mathrm{D}$ larger dipole moment for a water monomer in a cluster, than those based on the Bader-type analysis ${ }^{21,22}$. The aim of our work to generalize this approach to the use of atom-centered basis sets and to see what conclusions can be drawn on the basis of the "Hilbert-space analysis" utilizing the assignment of the basis functions to the individual atoms (and thus to the individual monomers). As will be seen, this analysis permits also to propose extremely simple criteria for identifying individual hydrogen bonds in a cluster, simpler than used in the literature ${ }^{23-30}$. In our study we will not pursue numerical accuracy but rather wish to obtain qualitative understanding, which may be achieved by performing not too sophisticated calculations.

In the recent work ${ }^{31}$ Torii investigated in a somewhat complicated manner the dynamic and static dipole moments of the water. As will be seen, his conclusion about the role of charge transfer effects in enhancement of static dipoles is completely in line with our results obtained in a quite different formalism.

\section{The calculations}

When one performs a supermolecule type quantum chemical calculation for a water cluster, then all the electrons are considered as part of the composite system, without assigning them to the different monomers. If we are interested in the dipole moments of individual monomers, then the overall electronic charge should be decomposed into parts in such a manner that each water molecule is assigned ten electrons. In the general case, when electron correlation is taken into account, such a decomposition of the electronic 
density may be rather problematic. However, if one uses a closed shell SCF method (Hartree-Fock or density functional theory, DFT) then this task can easily be accomplished by assigning five doubly filled localized molecular orbitals (LMO-s) to each water monomer. (Canonical orbitals are delocalized, so are not adequate for the purpose.) Then one has to attribute the electron density corresponding to these five localized orbitals to the given monomer, and calculate the dipole moment with these orbitals in the same manner as one calculates the dipole moment of a single molecule. Of course, one should take into account that the localized orbitals are not strictly restricted to the basis orbitals of the given monomer, so the basis set of the whole cluster should be used.

It is known that localized orbitals are not uniquely determined but depend on the localization criterion used. For that reason, we have used four quite different localization procedures. Two of them were standard ones: Boys localization ${ }^{32}$ and calculation of the "natural localized orbitals" (NLOs) of Weinhold group ${ }^{33}$. The other two represented generalizations of our approach applied for determining effective atomic orbitals ${ }^{34-37}$. In one of them the Magnasco-Perico (Mag.-Per.) localization criterion ${ }^{38}$ is applied to the individual monomers; it consists in maximizing the norm of the intramonomer part of the LMO-s. (It is Mulliken's net population generalized to an individual monomer.) The fourth scheme is the 3-dimensional "fuzzy atoms" analogue of the previous one: the intramonomer part of the LMO$\mathrm{s}$ is maximized in the part of the 3-dimensional space that has no sharp boundaries but is defined by the sum of the three atomic weight functions of Becke-type ${ }^{39,40}$, corresponding to one oxygen and two hydrogens of the monomer in question. Two of these localization criteria - the Boys-type and the fuzzy one-are based on criteria related to the 3D space, the other two use methods of the Hilbert space analysis, in which an individual monomer is identified with its three nuclei and the basis orbitals centered on them.

The Boys-localization and the calculation of NLO-s are standard procedures, we need not to discuss them here in any detail. In our schemes we are looking for a unitary transformation of the occupied molecular orbitals (MO-s)

$$
\psi_{i}=\sum_{j}^{o c c .} U_{j i} \varphi_{j},
$$

that permits to make maximal (or at least stationary) the norm of intramonomer part of the resulting transformed MO $\psi_{i}$ in either of the two schemes mentioned:

$$
\delta \frac{\left\langle\psi_{i}^{X} \mid \psi_{i}^{X}\right\rangle}{\left\langle\psi_{i} \mid \psi_{i}\right\rangle}=0 .
$$


Here superscript "X" denotes the intramonomer part of the orbital $\psi_{i}$.

The basis expansion of the orbital is, according to the transformation (1) is given by the coefficients $C_{\mu i}^{\text {loc. }}$ :

$$
\psi_{i}=\sum_{\mu} C_{\mu i}^{\text {loc. }} \chi_{\mu}=\sum_{\mu} \sum_{j}^{o c c .} C_{\mu j} U_{j i} \chi_{\mu},
$$

where the $C_{\mu j}$-S are the canonical orbital coefficients obtained in the SCF calculation.

If the Magnasco-Perico localization is used, then the intramonomer function $\psi_{i}^{X}$ is obtained by conserving only the basis orbitals located on the given monomer $X$ :

$$
\psi_{i}^{X}=\sum_{\mu \in X} C_{\mu i}^{l o c .} \chi_{\mu}=\sum_{\mu \in X} \sum_{j}^{o c c .} C_{\mu j} U_{i j} \chi_{\mu} .
$$

In the 3-dimensional fuzzy atoms analysis $\psi_{i}^{X}$ becomes

$$
\psi_{i}^{X}(\vec{r})=w_{X}(\vec{r}) \psi_{i}(\vec{r}) .
$$

Here $w_{X}(\vec{r})$ is the weight function assigned to the monomer X; it is calculated as the sum of the weight functions for the constituting atoms, calculated according to Ref. 40. (The weight functions of all the monomers sum to one in every point of the 3 -dimensional space.)

As discussed in Refs. 34-37, the variational condition (2) is fulfilled if the unitary matrix $\mathbf{U}$ used in the transformation (1) diagonalizes matrix $\mathbf{Q}$ with the elements

$$
Q_{i j}=\left\langle\psi_{i}^{X} \mid \psi_{j}^{X}\right\rangle
$$

These calculations have been performed by suitably modifying our programs ${ }^{34,36}$ devoted for calculating effective AO- $\mathrm{s}^{41}$.

For each monomer one obtains, in principle, as many transformed MO-s as is the total number of MO-s in the cluster. Out of them, we conserve only those five that are almost entirely localized on the given monomer and repeat the procedure for each monomer. The localized MO-s obtained for the same monomer are orthonormalized (every matrix $\mathbf{U}$ is unitary), but the MO-s localized on different monomers are not strictly orthogonal ${ }^{34}$. To fulfill the requirement that the dipole moments of the monomers should sum to the dipole moment of the cluster, the localized MO-s obtained are subjected to a symmetric Löwdin-orthogonalization before the dipole moments of the individual monomers is calculated.

An interesting property of the LMO-s obtained by these procedures is that-before the global orthogonalization mentioned is performed - their intramonomer parts also form an orthogonal $\operatorname{set}^{34-37}$. The five of these orbitals 
corresponding to the largest eigenvalues of matrix $\mathbf{Q}$ can be called the effective MO-s of the individual monomers: these are the orbitals by which the monomer is mainly participating in forming the cluster's wave function.

When considering the degree of localization/delocalization of a given localized orbital, we have calculated the quantities corresponding to Mulliken's net orbital populations. Actually the degree of the localization of the localized orbital $\psi_{i}$ on the "own" monomer A was calculated as

$$
l_{i}^{A}=\left\langle\psi_{i}^{A} \mid \psi_{i}^{A}\right\rangle=\sum_{\mu, \nu \in A} C_{\mu i}^{l o c * *} S_{\mu \nu} C_{\nu i}^{l o c .}
$$

while the delocalization to the given monomer was simply characterized by the sum of the squares of the coefficients.

We have dealt with relative large water clusters for which geometry optimization was affordable only by the not too big $6-311 \mathrm{G}(\mathrm{d}, \mathrm{p})$ basis set, but

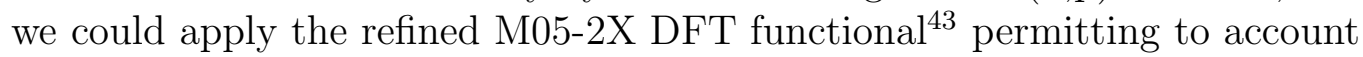
indirectly for correlation effects. As we are interested in qualitative, rather than quantitative effects, we have used these geometries also for the "single point" calculations by using the larger basis sets cc-pVTZ and aug-cc-pVTZ . The Boys-localized orbitals and the NLO-s were calculated by the Gaussian09 program system ${ }^{44}$, the other two types of LMO-s by suitable modifications of our programs used in ${ }^{34-37}$ : instead of a single atom a reference unit was a monomer. The LMO-s were outputted and sorted in each case and then read back to Gaussian-09. (For technical details we refer to the Supplementary material.)

Besides monomer and dimer calculations, we have considered all the four localization schemes for water clusters consisting of $6,10,20$ and 28 water molecules. Their structure has been taken from the literature ${ }^{45}$ and the geometries were reoptimized with the DFT functional and basis set indicated above. They are depicted on Figure 1; the actual Cartesian coordinates are given in the Supplementary material. Additionally, the calculations based on the Magnaso-Perico localization criterion have been performed for one cluster with every number of water molecules between 2 and 30. Their geometries were determined in the same manner as for those for the clusters discussed in detail. (The Cartesian coordinates are also given in the Supplementary material.)

\section{Results and discussion}

The dipole moment of a water monomer calculated with the functional M05$2 \mathrm{X}$ is $2.141 \mathrm{D}, 1.996 \mathrm{D}$ and $1.922 \mathrm{D}$ for the basis sets $6-311 \mathrm{G}(\mathrm{d}, \mathrm{p})$, cc-pVTZ and aug-cc-pVTZ, respectively; the last one does not differ significantly from 
the experimental value ${ }^{5}$ of $1.855 \mathrm{D}$. It is important to stress that this variation with the basis set does not influence the qualitative conclusions we could drawn by comparing the results obtained for different water clusters obtained by using the same basis set.

The dipole moment of a water dimer has been obtained 2.797 D, 2.651 D and $2.588 \mathrm{D}$ for the three basis sets mentioned. Table 1 . shows how it is composed of the dipole moments of the individual monomers according to the four different localization schemes (columns denoted "Fuzzy" to "Mag.-Per."). The dipole moments of the two monomers are not collinear and the resulting dipole moment is their vectorial sum, so the individual Cartesian components (but not the absolute values) of the monomer moments sum to the respective components of the dimer. In the case of all larger clusters studied, the overall dipole moment has also been always recovered within a reasonable numerical accuracy for all the localization schemes if a complete set of orthonormalized localized orbitals has been considered. However, when the LMO-s were truncated to the individual monomers, the vectorial sum of the monomers' dipole moments ceased to sum to that of the cluster.

Looking at the results, we see that the method of localization is practically irrelevant. This is the case in all calculations discussed in the present work; that is very important as to the reliability of the conclusions drawn. The numerical values indicate that the collective effects that lead to the increase of the dipole moments of individual water monomers in bulk water - although to a smaller extent - already manifest in the dimer case, too.

The last columns in the Tables contain the dipole moments calculated in the following manner. As have mentioned above, the intramonomer parts of the localized orbitals obtained by using the Magnasco-Perico localization criterion (i.e., maximizing the norm of the intramonomer parts of the LMO-s) form an orthogonal set of effective monomer orbitals, characterizing their internal electronic structure. ${ }^{34,35,37}$ One can renormalize these effective monomer orbitals, fill them by two electron each, and calculate the dipole moment of the individual monomers; this means omitting completely the delocalization tails of the LMO-s. We shall call this type of technique as Magnasco-Perico truncated (Mag.-Per.-trunc.) scheme. These values give a sort of "internal" or "intrinsic" dipole moments of the individual monomers in the given environment, as orbitals centered on the other monomers do not contribute to them. These "intrinsic" dipole moments in all cases, except the dimer, are significantly lower than those calculated by using the full localized orbitals. (We shall return to that point.)

In order to check that these results are not due to the specific localization scheme applied, we have performed the following alternative procedure, also giving internal dipole moments of monomers, although they are a little less 
well-cut conceptually. We have cut the LMO-s obtained by using the Boys procedure for a given monomer to the basis of that monomer, performed a Löwdin-orthonormalization of the orbitals obtained for each monomer separately, and calculated the dipole moments. The results obtained are very close to those in the last columns of Tables, the maximal difference being 0.03 D. ${ }^{46}$. (The actual values are listed in the Supplementary material.)

In the case of the water dimer the second monomer represents the hydrogen donor; its dipole moment does not practically change if the delocalization tails are omitted; the increase of its dipole moment should be attributed to the polarization due to the partner molecule. (The change of the geometry of this monomer in the dimer does not explain the effect, as it leads to a change in dipole moment only in the second decimal digit - cf Ref. 13.) However, cutting the delocalization tails for the hydrogen acceptor molecule reduces the dipole moment to a value close to that of the free monomer. ${ }^{47}$ We can conclude, therefore, that the dipole moment increase in the water clusters is due to two different mechanisms: the polarization and the delocalization effects - though their distinction may be difficult in the Hilbert space analysis if large basis sets with diffuse functions are used. Then turning to "3D analysis" may be desirable. (For the larger clusters studied below every monomer acts both as hydrogen acceptor and donor, so the behaviour of the proton acceptors and proton donors cannot be considered for them separately.)

Tables 2 to 5 contain the dipole moments of the individual water monomers calculated by the four localization schemes for the water clusters consisting of $6,10,20$ and 28 water molecules, respectively. For the smallest cluster the data obtained with the three different basis sets are displayed, for the larger ones those for 6-311G(d,p) basis set. (Some data for other basis sets are included in the "Supplementary material".) The results are quite insensitive to the method of localization in all cases without exception. Also, it can be seen that the average monomer's dipole moments exhibits a gradual increase with the increasing cluster size. Furthermore, the dipole moments of the monomers are significantly reduced if the delocalization tails of the MO-s are cut, and become close to the values usually given by the Bader-type analysis. However, the dipole moments obtained by cutting the delocalization tails of the MO-s do not sum to that of the overall cluster. This means that the dipole moment of a water cluster cannot be fully recovered in any calculation in which the individual water monomers are treated as spatially separated entities consisting of the three nuclei and ten electrons. As the delocalization between the monomers is essential for recovering the overall dipole moment of the whole system, we must accept that the individual water monomers can be delimited only in a quite fuzzy manner, and the electronic charge density in a given point cannot be assigned completely 
to that or another molecule. (The situation becomes even more involved if electron correlation is explicitly taken into account.) That means that the concept of a molecule in a water cluster - or water medium - is necessarily a fuzzy one, except if we restrict ourselves to considerations based merely on the position of the nuclei.

This is further illustrated by Figure 2, on which the average dipole moments of the water monomers and their mean square deviations are depicted as functions of the number of water monomers changing from 2 to 30. The upper curve is the dipole moment obtained with the "full" Magnasco-Perico localized orbitals containing the delocalization "tails", while one of the lower ones shows those obtained after cutting the "outside" parts of the LMO-s. One can see that both curves approach saturation around $n=15$ (Mag.-Per. $\approx 3.15 \mathrm{D}$, Mag.-Per.-trunc. $\approx 2.7 \mathrm{D})$, so the resulting effect of the tails is $c a$. $0.45 \mathrm{D}$. (The limiting value of $\approx 3.15 \mathrm{D}$ is roughly as much larger than the experimental value $\approx 2.8-2.95 \mathrm{D}$ as the difference seen for the free monomer, so our results seem not conflict with the experimental ones ${ }^{48}$. There is a third, independent, curve on this figure, too, showing the dipole moments calculated by using the Bader-type analysis. The closeness of the two lower curves is striking.

The comparison of the truncated and "full" Magnasco-Perico results are in agreement with the difference of ca. 0.5 D between the Bader and Wannier results for bulk water mentioned above, in complete agreement with the fact that the Wannier orbitals obtained in the plane wave calculations are essentially a generalization of the Boys-localized orbitals including the delocalization tails. These data show that the effect of the intermolecular delocalizations in the water clusters contributes ca. $0.45-0.5 \mathrm{D}$ per molecule to the resulting dipole moment.

The delocalization effects discussed are not some random ones. Significant delocalization is always observed within a hydrogen bond - the lone pair of the proton-acceptor molecule exhibits a delocalization to the molecule by which it forms a hydrogen bond. The importance of charge transfer effects in forming hydrogen bonds is well established in the literature ${ }^{49-59}$. The delocalization is mainly expected ${ }^{60-61}$ onto the $\sigma^{*}$ orbital of the latter molecule. Therefore, by studying the tails of the LMO-s, one can identify the pairs of hydrogen bonded molecules. Figure 3 displays the measures of delocalization for a pair of water molecules (the Mulliken net population on a given molecule, originating from the tails of those two localized orbitals centered on the partner molecule that are most delocalized) as the function of the O...O distance, for the cluster of 28 water molecules, and for all the four localization schemes considered. It can be seen that significant values occur only for the molecules with the O...O distances around 2.6-2.8 $\AA$, character- 
istic for the hydrogen bonded pairs. As only the proton-acceptor molecule of a given pair exhibits a significant delocalization, every H-bonded pair does also contribute a point near the zero level on this figure, showing the lack of the delocalization from the proton donor to the proton acceptor. The significant gap observed on this figure is in agreement with the fact that all the delocalization tails exceeding 0.03 correspond to hydrogen bonds also on the basis of their geometries ${ }^{62}$. That means that the existence or absence of the hydrogen bond between a pair of water molecules in a water cluster can be discriminated using the delocalization.

It is interesting to note that the Magnasco-Perico delocalization parameter displayed on Figure 3 is 0.048 at the equilibrium geometry of a single water dimer, which is less than the value for most of hydrogen bonded pairs in the actual cluster. This means that in this respect we have again met a typical collective effect - similar to those discussed in our recent paper Ref. 13. The same is observed for the dipole moments, too. This indicates again that the study of a hydrogen bonded dimer system may be not sufficiently relevant for discussing the properties of a bigger cluster.

The investigation of the localization patterns permits also to distinguish between classes of water molecules differing by the number of hydrogen bonds in which they are proton donors and proton acceptors. Figure 4 displays the Mulliken net populations on the "own" molecule for each of the five localized orbitals corresponding to the individual water monomers in the $\left(\mathrm{H}_{2} \mathrm{O}\right)_{28}$ cluster. (The case of using the NLO localized orbitals is shown, the other three are quite analogous.) One orbital - corresponding to the oxygen $1 s$ one - is always perfectly localized on the given molecule; two orbitals show a Mulliken net population slightly exceeding one (for them the overlap populations with the other molecules give some negative contributions), while there are two orbitals with net populations lower than one. It can be seen that the number of orbitals with localization lower than $\sim 0.98$ equals the number of hydrogen bonds in which the given molecule is a proton acceptor. As a consequence, especially those water molecules show a characteristic localization pattern, that have one hydrogen bond in which they are proton acceptors and two ones in which they are proton donors (molecules of 1A:2D type).

The differences in the delocalization patterns manifest also in the values of dipole moments of water molecules in different hydrogen bonded environment. Table 6 compares the average dipole moments calculated for the complexes with 26-30 water molecules for Magnasco-Perico localization criterion without and with truncation. It can be seen that the average dipole moments are the largest for waters of type "two acceptors-two donors" (2A:2D). Similar conclusion has been obtained by Akase and Aida ${ }^{63}$ who used the TRM2-R 
polarisable model ${ }^{64}$ for small water clusters, as well as in our recent work ${ }^{13}$ by using the Bader-type dipole moments. It is interesting that the order of dipole moments for 1A:2D and 2A:1D type waters is reversed if the tails of the LMO-s is cut. That indicates how large is the importance of different fine quantum mechanical effects in determining collective effects in water medium.

When discussing delocalization effects, one should stress - as we do it for decades $\left(e . g .^{65-67}\right.$ — the distinction between the "mathematical" and "physical" delocalizations. The former lead to the "orthogonalization tails" which are solely due to the fact that it is more convenient to work with orthonormalized MO-s than with non-orthogonal ones, while the latter reflect the actual physical interactions in the system. In order to show that the delocalizations discussed in the present work do represent true physical effects characteristic for the hydrogen bonds, we have performed the following procedure. The monomer MO-s, obtained by using the Magnasco-Perico localization criterion and truncated in order to calculate the "intrinsic" monomer dipole moments shown in the last columns of Tables, represent orthogonal sets for the given monomer, but the orbitals of the different monomers are not orthogonal to each other. Performing the Löwdin orthonormalization of these truncated orbitals, and calculating dipole moments of each monomer by using its five globally orthogonalized orbitals, we can account for the effects of the "mathematical" delocalizations. We have found (the actual numbers are listed in the Supplementary material) that the dipole moments in all but one cases give somewhat larger dipole moments than those obtained for the strictly intramonomer orbitals, but the effect is rather small - it does not exceed $0.08 \mathrm{D}$ - so the results remained significantly smaller than those in the first four columns. (The single exception was the proton acceptor monomer in the dimer case, for which the mathematical delocalization lead to a slight decrease of the dipole moment.) This comparison indicates that the effects of delocalizations along the hydrogen bonds reflect essential physical aspects of the water structure.

The whole situation can be concisely characterized by the following three figures, showing some distributions obtained by using the Magnasco-Perico localization criterion.

Figure 5 shows the distribution of the measure of the delocalization tails from the proton acceptor to the proton donor molecules for the $\left(\mathrm{H}_{2} \mathrm{O}\right)_{30}$ cluster, It can be seen, that for most hydrogen bonded pairs it exceeds the value characteristic for a water dimer, indicated by a vertical bar. That is a pronounced manifestation of the collective effects characteristic for water clusters. This figure is quite analogous to the distribution of the intermolecular bond orders obtained most recently ${ }^{13}$, which is in agreement with the close 
connection between these quantities. Figure 6 shows the distribution of the dipole moments calculated for the individual water monomers in all clusters with 25 to 30 water molecules. All the dipole moments are significantly larger that in the monomers or dimers, which is due to the larger delocalizations and the mutual polarizations - the latter is also a collective effect. Figure 7 shows the analogous distribution for the "intrinsic" dipole moments, obtained by cutting the delocalized tails of the MO-s and renormalizing them. It can be seen that the dipole moments are significantly reduced as compared with the previous figure, but they are still much larger than the monomer

and dimer values. It may be of interest to note that the last distribution is pretty similar to the analogous one recently obtained ${ }^{13}$ for the Bader-type analysis, indicating that the low dipole values given by the latter are indeed due to the inability of that scheme to account for delocalization effects.

We may conclude that there are two physical effects influencing the dipole moments of the water monomers in clusters (or bulk water): mutual polarization and delocalization, which may have an important message for developing new force fields. Although both of them trend to increase the dipole moments of the individual monomers, their effect is not strictly additive; for instance, the truncation of the localized MO-s for the proton acceptor monomer in the water dimer does increase - and not decrease - its dipole moment if basis sets without diffuse functions are used.

\section{Summary}

We have computed the dipole moments of the individual water molecules in different water clusters by calculating localized molecular orbitals and assigning five localized orbitals to each water monomer unit. Using four quite different localization schemes, all the localized orbitals were found to be indeed almost completely situated on the respective molecules, so their delocalization tails were small. Nonetheless, these small delocalization tails are of decisive importance for reproducing the collective effects observed in water and determining the dipole moments of the individual monomers: cutting them one gets significantly lower dipole moments, comparable to those one obtains in the framework of Bader analysis. This shows that the notion that fluid or solid water consists of well-distinguishable water molecules has some limitations: the individual molecules can be delimited only in a quite fuzzy manner. The analysis of the tails of the localized orbitals can also be used to identify the pairs of water molecules actually forming hydrogen bonds. 


\section{Supporting information}

The Supporting Informations contain

1. the coordinates and figures of the optimized structures of all the complexes studied;

2. the Gaussian-09 control cards used for calculating the dipole moments corresponding to the monomers with the respective localized orbitals;

3 . the dipole moments of the individual water monomers in the clusters of $2,6,10,20$ and 28 water molecules, calculated by using seven different localization schemes at the M052X/6-311G(d.p level.

\section{Acknowledgment}

The authors acknowledge the partial financial support of the Hungarian Scientific Research Fund (grant OTKA K108721). 


\section{References}

1. The experimental dipole moments in the bulk water were obtained in Xray diffraction experiments by using standard atomic form factors and standard rigid geometry of the water molecules, by adjusting the effective atomic charges to reproduce the experimental diffraction pattern. This approach assumes the individual water molecules to be neutral. As will be seen, our approach is more general than that model and may even motivate some refinements in processing the experimental data.

2. Coulson, C.A.; Eisenberg, D. Interactions of H2O Molecules in Ice. II. Interaction Energies of $\mathrm{H} 2 \mathrm{O}$ Molecules in Ice. Proc. $R$. Soc. London 1966, A 291, 454-459.

3. Badyal, Y.S.; Saboungi, M.-L.; Price, D.L.; Shastri, S.D.; Haeffner, D.R.; Soper, A.K. Electron Distribution in Water. J. Chem. Phys. 2000, 112, 9206-9208.

4. Brookes, D.H.; Head-Gordon, T. Family of Oxygen-Oxygen Radial Distribution Functions for Water J. Phys. Chem. Lett. 2015, 6, 2938-2943.

5. Clough, S.A.; Beers, Y.; Klein, G.P.; Rothman, L.S. Dipole Moment of Water from Stark Measurements of H2O, HDO, and D2O. J. Chem. Phys. 1973, 59, 2554-2559.

6. Gregory, J. K.; Clary, D. C.; Liu, K.; Brown, M. G. ; Saykally, R. J. The Water Dipole Moment in Water Clusters. Science 1997, 275, $814-817$.

7. Laasonen, K.; Sprik, M.; Parrinello, M.; Car, R. Ab Initio Liquid Water. J. Chem. Phys. 1993, 99, 9080-9089.

8. Chalmet, S.; Ruiz-López, M.F. The Reaction Field of a Water Molecule in Liquid Water: Comparison of Different Quantum/Classical Models. J. Chem. Phys. 2001, 115 5220-5227.

9. Millot, C.; Costa Cabral, B. J. Electronic Properties of Liquid Water by Sequential Molecular Dynamics/Density Functional Theory. Chem. Phys. Letters, 2008, 460, 466-469.

10. Kongsted, J.; Osted, A,; Mikkelsen, K.V.; Christiansen, O. Dipole and Quadrupole Moments of Liquid Water Calculated Within the Coupled Cluster/Molecular Mechanics. Chem. Phys. Letters, 2002, 364, 379386.

11. Georg, H.C.; Canuto S. A Sequential QM/MM Study Using the Free Energy Gradient Method. J. Phys. Chem. B, 2012, 116, 11247-11254. 
12. Willow, S. Y.; Salim, M. A.; Kim, K. S.; Hirata, S. Ab Initio Molecular Dynamics of Liquid Water using Embedded-Fragment Second-Order Many-Body Perturbation Theory towards its Accurate Property Prediction. Sci. Rep. 2015, 5, 14358.

13. Bakó I.; Mayer I. Hierarchy of the Collective Effects in Water Clusters. J. Phys. Chem. A, 2016, 120, 631-638.

14. Bader, R.F.W. Atoms in Molecules: A Quantum Theory (Oxford University Press, Oxford, 1990).

15. Bader, R.F.W.; Matta, C.F. Properties of Atoms in Crystals: Dielectric Polarization. Int. J. Quantum Chem. 2001, 85, 592-607.

16. Gubskaya, A. V.; Kusalik, P. G. The Total Molecular Dipole Moment for Liquid Water. J. Chem. Phys. 2002, 117, 5290-5303.

17. Silvestrelli, P. L.; Parrinello, M. Water Dipole Moment in the Gas and Liquid Phase. Phys. Rev. Lett. 1999, 82, 3308-3311.

18. Dyer, P.J.; Cummings, P. Hydrogen Bonding and Induced Dipole Moments in Water: Predictions from Gaussian Charge Polarisable Model and Car-Parrinello Molecular Dynamics. J. Chem. Phys. 2006, 125, 144519 .

19. Zhang, C.; Donadio, D.; Gygi, F.; Galli, G. First Principles Simulations of the Infrared Spectrum of Liquid Water using Hybrid Density Functionals. J. Chem. Theory Comput. 2011, 7, 1443-1449.

20. Sharma, M,; Resta, R; Car R. Dipolar Correlations and the Dielectric Permittivity of Water. Phys. Rev. Lett. 2007, 98, 247401.

21. Batista, E.R.; Xantheasa, S.S.; Hannes Jonsson, H. Electric Fields in Ice and Near Water Clusters. J. Chem. Phys. 2000, 112, 3285-3292.

22. Delle Site, L.; Alavi, A.; Lynden-Bell, R.M. The Electrostatics Properties of Water Molecules in Condensed Phases: an ab Initio Study. Mol. Phys. 1999, 96, 1683-1693.

23. Reiher, M.: Kirchner, B. A Wavefunction-Based Criterion for the Detection of Intermolecular Interactions in Molecular Dynamics Simulations. J. Phys. Chem. A, 2003, 107, 4141-4146.

24. Thar, J.; Kirchner, B. Hydrogen Bond Detection. J. Phys. Chem. A, 2006, 110, 4229-4237.

25. Ehrhardt, C.; Ahlrichs R. Population Analysis Based on Occupation Numbers II. Relationship Between Shared Electron Numbers and Bond Energies and Characterization of Hypervalent Contributions. Theor. Chim. Acta. 1985, 68, 231-245.

26. Popelier, P. Atoms in Molecules. An Introduction. (Prentice-Hall, Harlow, 2000). 
27. Poater, J.; Fradera, X,; Sola, M.; Duran, M.; Simon, S. On the Electron-Pair Nature of the Hydrogen Bond in the Framework of the Atoms in Molecules Theory. Chem. Phys. Letters, 2003, 369, 248255.

28. Koch, U.; Popelier, P.L.A. Characterization of C-H-O Hydrogen Bonds on the Basis of the Charge Density. J. Phys. Chem. 1995, 99, 97479754.

29. Grabowski, S.J. Hydrogen Bonding Strength - Measures Based on Geometric and Topological Parameters. J. Phys. Org. Chem. 2004, $17,18-31$.

30. Grabowski, S.J. What Is the Covalency of Hydrogen Bonding? Chem. Rev. 2011, 111, 2597-2625.

31. Torii, H. Extended Nature of the Molecular Dipole of Hydrogen Bonded Water. J. Phys. Chem A, 2014, 117, 2044-2051.

32. Boys, S.F. Construction of Some Molecular Orbitals to Be Approximately Invariant for Changes from One Molecule to Another. Rev. Mod. Phys. 1960, 32, 296-299.

33. Reed A.E.; Weinhold F. Natural Localized Molecular Orbitals. J. Chem. Phys. 1985, 83, 1736-1740.

34. Mayer, I, Non-orthogonal Localized Orbitals and Orthogonal Atomic Hybrids Derived from Mulliken's Population Analysis. Chem. Phys. Letters 1995, 242, 499-506.

35. Mayer, I. Atomic Orbitals from Molecular Wave Functions: the Effective Minimal Basis. J. Phys. Chem. 1996, 100, 6249-6257.

36. Mayer I.; Salvador, P. Effective Atomic Orbitals for Fuzzy Atoms. J. Chem. Phys. 2009, 130, 234106.

37. Mayer, I. Effective Atomic Orbitals: A Tool for Understanding Electronic Structure of Molecules Int. J. Quantum Chem. 2014, 114, 1041-1047.

38. Magnasco V.; Perico A. Uniform Localization of Atomic and Molecular Orbitals. I. J. Chem. Phys. 1967, 47, 971-981.

39. Becke, A.D. A Multicenter Numerical Integration Scheme for Polyatomic Molecules. J. Chem. Phys. 1988, 88, 2547-2553.

40. Mayer, I.; Salvador, P. Overlap Populations, Bond Orders and Valences for "Fuzzy" Atoms. Chem. Phys. Letters 2004, 383, 368-375.

41. In the Magnasco-Perico case there is a cheaper, but fully equivalent, algorithm, too, using the density matrix ${ }^{34}$. It is used in our free program $^{42}$ for calculating effective AO-s, and has been utilized, here, too. That difference, however, does not have any significance. 
42. Mayer, I. Program EFF-AO, Budapest, 2008. May be downloaded from the web-site http://occam.ttk.mta.hu

43. Zhao, Y.; Schultz, N.E.; Truhlar D.G. Design of Density Functionals by Combining the Method of Constraint Satisfaction with Parametrization for Thermochemistry, Thermochemical Kinetics, and Noncovalent Interactions. J. Chem. Theory and Comput., 2006, 2, 364-382.

44. Frisch, M. J.; Trucks, G. W.; Schlegel, H. B.; Scuseria, G. E.; Robb, M. A.; Cheeseman, J. R.; Scalmani, G.; Barone, V.; Mennucci, B.; Petersson, G. A.; Gaussian 09, revision D.01; Gaussian, Inc.; Wallingford, CT, 2013.

45. Qian, P; Song, W.; Lu, L.; Yang, Y. Ab Initio Investigation of Water Clusters $(\mathrm{H} 2 \mathrm{O}) \mathrm{n}(\mathrm{n}=2-34)$. Int. J. Quantum Chem. 2010, 110, 1923-1937.

46. The numbers obtained by this procedure always are slightly lower than those in the last columns of the Tables. One can hardly attribute any significance to this observation.

47. This latter observation does not hold to the aug-cc-pVTZ basis (basis C), indicating once again that basis sets with diffuse basis functions are not really suitable for "Hilbert space" analysis.

48. This is the case irrespective of the limitations inherent in the experimental data analysis procedure, mentioned above in ${ }^{1}$.)

49. Khaliullin, R.Z.; Cobar, E.A.; Lochan, R.C.; Bell, A.T.; Head-Gordon, M. Unravelling the Origin of Intermolecular Interactions Using Absolutely Localized Molecular Orbitals. J. Phys. Chem. A, 2007, 111, 8753-8765.

50. Kitaura, K.; Morokuma, K. A New Energy Decomposition Scheme for Molecular Interactions within the Hartree-Fock Approximation Int. J. Quantum Chem. 1976, 10, 325-340.

51. Chen, W.; Gordon, M. S. Energy Decomposition Analyses for ManyBody Interaction and Applications to Water Complexes J. Phys. Chem. 1996, 100, 14316-14328.

52. Bagus, P. S.; Hermann, K.; Bauschlicher, C. W. A New Analysis of Charge Transfer and Polarization for Ligand-Metal Bonding: Model Studies Al4CO and Al4NH3. J. Chem. Phys. 1984, 80, 4378-4386.

53. Glendening, R.E.D.; Streitwieser, A. Natural Energy Decomposition Analysis - An Energy Partitioning Procedure for Molecular Interactions with Application to Weak Hydrogen-Bonding, Strong Ionic, and Moderate Donor-Acceptor Complexes, J. Chem. Phys. 1994, 100, 2900-2909. 
54. Schenter, G. K.; Glendening, E. D. Natural Energy Decomposition Analysis: The Linear Response Electrical Self Energy, J. Phys. Chem. 1996, 100, 17152-17156.

55. Iwata, S. Analysis of Hydrogen Bond Energies and Hydrogen Bonded Networks in Water Clusters (H2O)20 and (H2O)25 Using the ChargeTransfer and Dispersion Terms. Phys. Chem. Chem. Phys. 2014, 16, $11310-11317$.

56. Iwata, S.; Bandyopadhyay, P.; Xantheas, S.S. Cooperative Roles of Charge Transfer and Dispersion Terms in Hydrogen-Bonded Networks of $(\mathrm{H} 2 \mathrm{O}) \mathrm{n}, \mathrm{n}=6,11$, and 16. J. Phys. Chem. A, 2013, 117, 66416651.

57. Khaliullin, R.Z.; Bell, A.T.; Head-Gordon M. Electron Donation in the Water-Water Hydrogen Bond. Chem. Eur. J. 2009, 15, 851-855.

58. Iwata, S. Energy Analysis of Weak Electron-Donor-Acceptor Complexes and Water Clusters with the Perturbation Theory Based on the Locally Projected Molecular Orbitals: Charge-Transfer and Dispersion Terms. Phys. Chem. Chem. Phys. 2012, 14, 7787-7794.

59. Su, P.; Jiang, Z.; Chen, Z.; Wu, W. Energy Decomposition Scheme Based on the Generalized Kohn-Sham Scheme J. Phys. Chem. A, 2014, 118, 2531-2542.

60. Weinhold, F. Nature of H-Bonding in Clusters, Liquids, and Enzymes: an ab Initio, Natural Bond Orbital Perspective. J. Mol. Struct. (Theochem) 1997, 398-399, 181-197.

61. Weinhold , F. Natural Bond Orbital Analysis: A Critical Overview of its Relationship to Alternative Bonding Perspectives. J. Comp. Chem. 2012, 33, 2363-2379.

62. There is a peculiar point with an $\mathrm{R}(\mathrm{O} \ldots \mathrm{O}) \sim 3.2 \AA$, with the delocalization parameter between 0.01 and 0.018 depending on the localization method. Its geometry can perhaps be identified with a dihydrogen bond.

63. Akase, D.; Aida, M. Distribution of Topologically Distinct Isomers of Water Clusters and Dipole Moments of Constituent Water Molecules at Finite Atmospheric Temperatures J. Phys. Chem. A, 2014, 118, 7911-7924.

64. Burnham, C. J.; Xantheas, S. S. Development of Transferable Interaction Models for Water. III. Reparametrization of an All-Atom Polarizable Rigid Model (TTM2-R) from First Principles. J. Chem. Phys. 2002, 116, 1500-1510.

65. Mayer, I. Non-orthogonal Localized Orbitals to Study Delocalization Effects. Chem. Phys. Letters 1982 89, 390-394. 
66. Mayer, I. Quantum Chemical description of the Chemical Bond (in Hungarian). DSc thesis, Hungarian Academy of Sciences, Budapest, 1986.

67. Asturiol, D.; Salvador, P.; Mayer, I. Dissecting the Hindered Rotation of Ethane. ChemPhysChem, 2009 10, 1987-1992. 
TABLE 1

Dipole moments of the individual water monomers in a water dimer, calculated by using the M05-2X DFT functional, four different localization schemes and three different basis sets A: 6-311G(d,p), B: cc-pVTZ, and C: aug-cc-pVTZ.

\begin{tabular}{|ccccccc|}
\hline Basis & Monomer & Fuzzy & Boys & NLO & Mag.-Per. & Mag.-Per. trunc. \\
\hline \multirow{2}{*}{ A } & 1 & 2.386 & 2.382 & 2.385 & 2.388 & 2.199 \\
& 2 & 2.348 & 2.349 & 2.345 & 2.341 & 2.334 \\
B & 1 & 2.230 & 2.226 & 2.231 & 2.230 & 2.015 \\
& 2 & 2.204 & 2.206 & 2.201 & 2.194 & 2.198 \\
C & 1 & 2.171 & 2.167 & 2.173 & 2.175 & 2.237 \\
& 2 & 2.157 & 2.159 & 2.153 & 2.146 & 2.075 \\
\hline
\end{tabular}

The total dipole moment of the overall cluster by using basis $\mathrm{A}$ is $2.797 \mathrm{D}$, after the truncation of the "tails" of the LMO-s the vectorial sum of the monomer dipoles is $2.482 \mathrm{D}$. 
TABLE 2

Dipole moments of the individual water monomers in a cluster of six water molecules, calculated by using the M05-2X DFT functional, four different localization schemes and three different basis sets A: 6-311G(d,p), B: cc-pVTZ, and C: aug-cc-pVTZ.

\begin{tabular}{|ccccccc|}
\hline \multirow{2}{*}{ Basis } & Monomer & Fuzzy & Boys & NLO & Mag.-Per. & Mag.-Per. trunc. \\
\hline \multirow{2}{*}{ A } & 1 & 2.928 & 2.926 & 2.919 & 2.923 & 2.488 \\
& 2 & 3.016 & 3.013 & 3.010 & 3.005 & 2.654 \\
& 3 & 3.163 & 3.164 & 3.152 & 3.150 & 2.659 \\
& 4 & 2.738 & 2.742 & 2.733 & 2.725 & 2.623 \\
& 5 & 2.790 & 2.791 & 2.784 & 2.781 & 2.505 \\
B & 6 & 2.714 & 2.717 & 2.708 & 2.704 & 2.420 \\
& 1 & 2.774 & 2.771 & 2.766 & 2.767 & 2.389 \\
& 2 & 2.905 & 2.902 & 2.898 & 2.898 & 2.544 \\
& 3 & 3.033 & 3.033 & 3.021 & 3.019 & 2.571 \\
& 4 & 2.611 & 2.614 & 2.606 & 2.601 & 2.496 \\
C & 5 & 2.666 & 2.667 & 2.662 & 2.660 & 2.394 \\
& 6 & 2.580 & 2.582 & 2.572 & 2.572 & 2.306 \\
& 1 & 2.720 & 2.717 & 2.708 & 2.725 & 2.608 \\
& 2 & 2.874 & 2.871 & 2.868 & 2.870 & 2.743 \\
& 3 & 2.989 & 2.990 & 2.978 & 2.962 & 2.815 \\
& 4 & 2.591 & 2.594 & 2.582 & 2.550 & 2.609 \\
& 5 & 2.635 & 2.635 & 2.630 & 2.621 & 2.619 \\
& 6 & 2.530 & 2.532 & 2.522 & 2.523 & 2.495 \\
\hline
\end{tabular}

The total dipole moment of the overall cluster by using basis $\mathrm{A}$ is $1.861 \mathrm{D}$, after the truncation of the "tails" of the LMO-s the vectorial sum of the monomer dipoles is $1.721 \mathrm{D}$. 
TABLE 3

Dipole moments of the individual water monomers in a cluster of ten water molecules, calculated by using the M05-2X DFT functional, four different localization schemes and 6-311G(d,p) basis set.

\begin{tabular}{|cccccc|}
\hline Monomer & Fuzzy & Boys & NLO & Mag.-Per. & Mag.-Per. trunc. \\
\hline 1 & 2.985 & 2.979 & 2.976 & 2.979 & 2.521 \\
2 & 3.119 & 3.107 & 3.113 & 3.108 & 2.712 \\
3 & 3.295 & 3.291 & 3.281 & 3.283 & 2.746 \\
4 & 3.167 & 3.165 & 3.154 & 3.153 & 2.653 \\
5 & 3.155 & 3.153 & 3.143 & 3.142 & 2.647 \\
6 & 3.111 & 3.099 & 3.104 & 3.100 & 2.717 \\
7 & 3.226 & 3.211 & 3.218 & 3.215 & 2.798 \\
8 & 3.239 & 3.238 & 3.226 & 3.223 & 2.705 \\
9 & 3.209 & 3.193 & 3.201 & 3.198 & 2.755 \\
10 & 2.899 & 2.897 & 2.892 & 2.883 & 2.613 \\
\hline
\end{tabular}

The total dipole moment of the overall cluster is $2.793 \mathrm{D}$, after the truncation of the "tails" of the LMO-s the vectorial sum of the monomer dipoles is $2.597 \mathrm{D}$. 
TABLE 4

Dipole moments of the individual water monomers in a cluster of twenty water molecules, calculated by using the M05-2X DFT functional, four different localization schemes and $6-311 \mathrm{G}(\mathrm{d}, \mathrm{p})$ basis set.

\begin{tabular}{|cccccc|}
\hline Monomer & Fuzzy & Boys & NLO & Mag.-Per. & Mag.-Per. trunc. \\
\hline 1 & 3.161 & 3.143 & 3.154 & 3.150 & 2.675 \\
2 & 3.325 & 3.303 & 3.316 & 3.316 & 2.782 \\
3 & 3.579 & 3.581 & 3.560 & 3.557 & 2.962 \\
4 & 3.257 & 3.255 & 3.243 & 3.242 & 2.733 \\
5 & 3.184 & 3.166 & 3.177 & 3.175 & 2.716 \\
6 & 3.521 & 3.489 & 3.510 & 3.514 & 2.856 \\
7 & 3.016 & 3.008 & 3.009 & 3.000 & 2.615 \\
8 & 3.012 & 3.006 & 3.003 & 3.007 & 2.543 \\
9 & 3.221 & 3.201 & 3.212 & 3.211 & 2.707 \\
10 & 3.377 & 3.375 & 3.362 & 3.363 & 2.812 \\
11 & 3.019 & 3.011 & 3.012 & 3.004 & 2.634 \\
12 & 3.506 & 3.475 & 3.496 & 3.499 & 2.847 \\
13 & 3.579 & 3.581 & 3.561 & 3.559 & 2.962 \\
14 & 2.986 & 2.979 & 2.979 & 2.970 & 2.621 \\
15 & 3.322 & 3.322 & 3.307 & 3.305 & 2.777 \\
16 & 3.289 & 3.266 & 3.279 & 3.278 & 2.734 \\
17 & 3.198 & 3.196 & 3.185 & 3.185 & 2.691 \\
18 & 3.217 & 3.214 & 3.202 & 3.203 & 2.695 \\
19 & 3.054 & 3.050 & 3.045 & 3.048 & 2.580 \\
20 & 3.040 & 3.035 & 3.031 & 3.034 & 2.567 \\
\hline
\end{tabular}

The total dipole moment of the overall cluster is $1.736 \mathrm{D}$, after the truncation of the "tails" of the LMO-s the vectorial sum of the monomer dipoles is $1.647 \mathrm{D}$. 
TABLE 5

Dipole moments of the individual water monomers in a cluster of twenty eight water molecules, calculated by using the M05-2X DFT functional, four different localization schemes and 6-311G(d,p) basis set.

\begin{tabular}{|cccccc|}
\hline Monomer & Fuzzy & Boys & NLO & Mag.-Per. & Mag.-Per. trunc. \\
\hline 1 & 3.461 & 3.462 & 3.445 & 3.454 & 2.993 \\
2 & 3.569 & 3.554 & 3.555 & 3.567 & 2.979 \\
3 & 3.281 & 3.273 & 3.272 & 3.270 & 2.749 \\
4 & 3.327 & 3.320 & 3.316 & 3.316 & 2.830 \\
5 & 3.325 & 3.316 & 3.316 & 3.311 & 2.842 \\
6 & 3.262 & 3.254 & 3.252 & 3.263 & 2.771 \\
7 & 3.312 & 3.305 & 3.302 & 3.301 & 2.801 \\
8 & 3.310 & 3.298 & 3.299 & 3.306 & 2.816 \\
9 & 3.307 & 3.300 & 3.297 & 3.294 & 2.817 \\
10 & 3.414 & 3.407 & 3.404 & 3.403 & 2.921 \\
11 & 3.239 & 3.231 & 3.230 & 3.227 & 2.776 \\
12 & 3.036 & 3.043 & 3.026 & 3.014 & 2.665 \\
13 & 3.246 & 3.241 & 3.237 & 3.231 & 2.835 \\
14 & 3.253 & 3.250 & 3.240 & 3.238 & 2.719 \\
15 & 3.325 & 3.315 & 3.310 & 3.316 & 2.770 \\
16 & 3.194 & 3.189 & 3.184 & 3.185 & 2.676 \\
17 & 3.238 & 3.234 & 3.225 & 3.224 & 2.709 \\
18 & 3.226 & 3.224 & 3.211 & 3.209 & 2.675 \\
19 & 3.178 & 3.173 & 3.167 & 3.166 & 2.679 \\
20 & 3.183 & 3.168 & 3.179 & 3.174 & 2.766 \\
21 & 3.150 & 3.148 & 3.140 & 3.138 & 2.657 \\
22 & 3.188 & 3.175 & 3.182 & 3.174 & 2.792 \\
23 & 3.194 & 3.179 & 3.188 & 3.182 & 2.776 \\
24 & 3.242 & 3.221 & 3.236 & 3.230 & 2.810 \\
25 & 3.147 & 3.132 & 3.141 & 3.135 & 2.729 \\
26 & 3.106 & 3.093 & 3.099 & 3.095 & 2.708 \\
27 & 3.018 & 3.004 & 3.013 & 3.008 & 2.651 \\
28 & 2.901 & 2.899 & 2.895 & 2.884 & 2.606 \\
\hline & & & & & \\
\hline
\end{tabular}

The total dipole moment of the overall cluster is $3.745 \mathrm{D}$, after the truncation of the "tails" of the LMO-s the vectorial sum of the monomer dippers is $3.651 \mathrm{D}$. 
TABLE 6

Average Dipole moments of the water monomers in different hydrogen bonded environment calculated for the clusters with 26 to 30 water molecules and the Magnasco-Perico (Mag.-Per.) localization scheme without and with truncation of the delocalization "tails". (6-311G** basis, M05-2X functional).

\begin{tabular}{|ccc|}
\hline Type & Mag.-Per. & Mag.-Per.-trunc. \\
\hline 1A:2D & 3.11 & 2.71 \\
2A:1D & 3.18 & 2.66 \\
2A:2D & 3.30 & 2.83 \\
\hline
\end{tabular}




\section{FIGURE CAPTIONS}

\section{Figure 1}

The water clusters with $6,10,20$ and 28 water molecules, considered.

\section{Figure 2}

Average dipole moments and their main square deviation of the water molecules as the function of the number of water molecules in the clusters studied, using the Magnasco-Perico localization criterion, without and with truncation of the "tails" of the LMO's, and the Bader method.

\section{Figure 3}

The measures of delocalization for a pair of water molecules (the Mulliken net population on a given molecule, originating from the tail of a localized orbital centered on the partner molecule) as function of the O...O distance. The curves are shown for four different orbital localization methods.

\section{Figure 4}

Mulliken net populations on the "own" molecule for each of the five NLO localized orbitals corresponding to the individual water monomers in the $\left(\mathrm{H}_{2} \mathrm{O}\right)_{28}$ cluster.

\section{Figure 5}

Distribution of the measure of the delocalization tails from the proton acceptor to the proton donor molecules calculated by using the Magnasco-Perico localization for the $\left(\mathrm{H}_{2} \mathrm{O}\right)_{30}$ cluster.

\section{Figure 6}

Distribution of the dipole moments of water molecules calculated by using the Magnasco-Perico localization for the individual water monomers in all clusters with 25 to 30 water molecules.

\section{Figure 7}

Distribution of the dipole moments of water molecules calculated by using the Magnasco-Perico localization and cutting the delocalization tails for the individual water monomers in all clusters with 25 to 30 water molecules. 

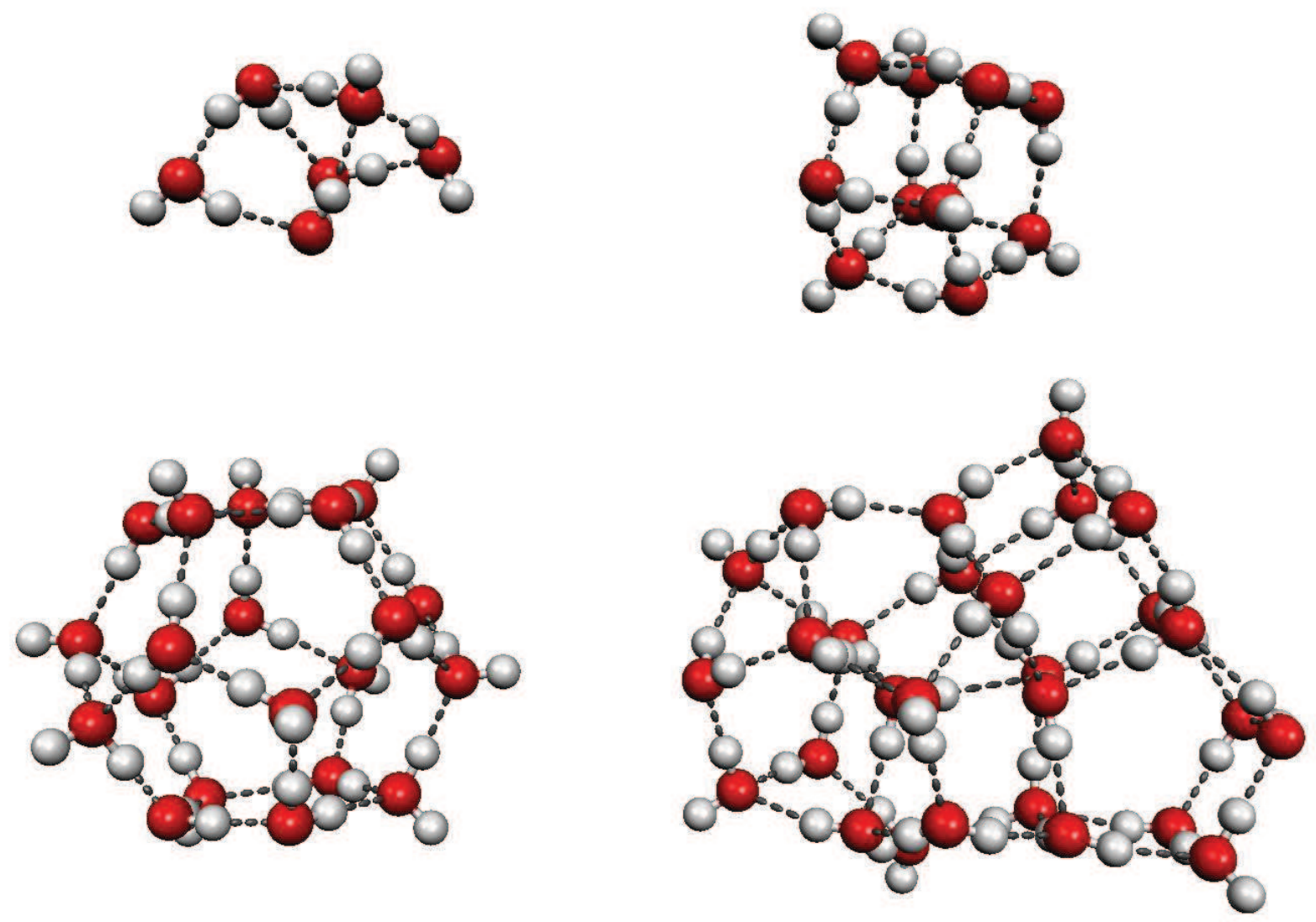

Figure 1 


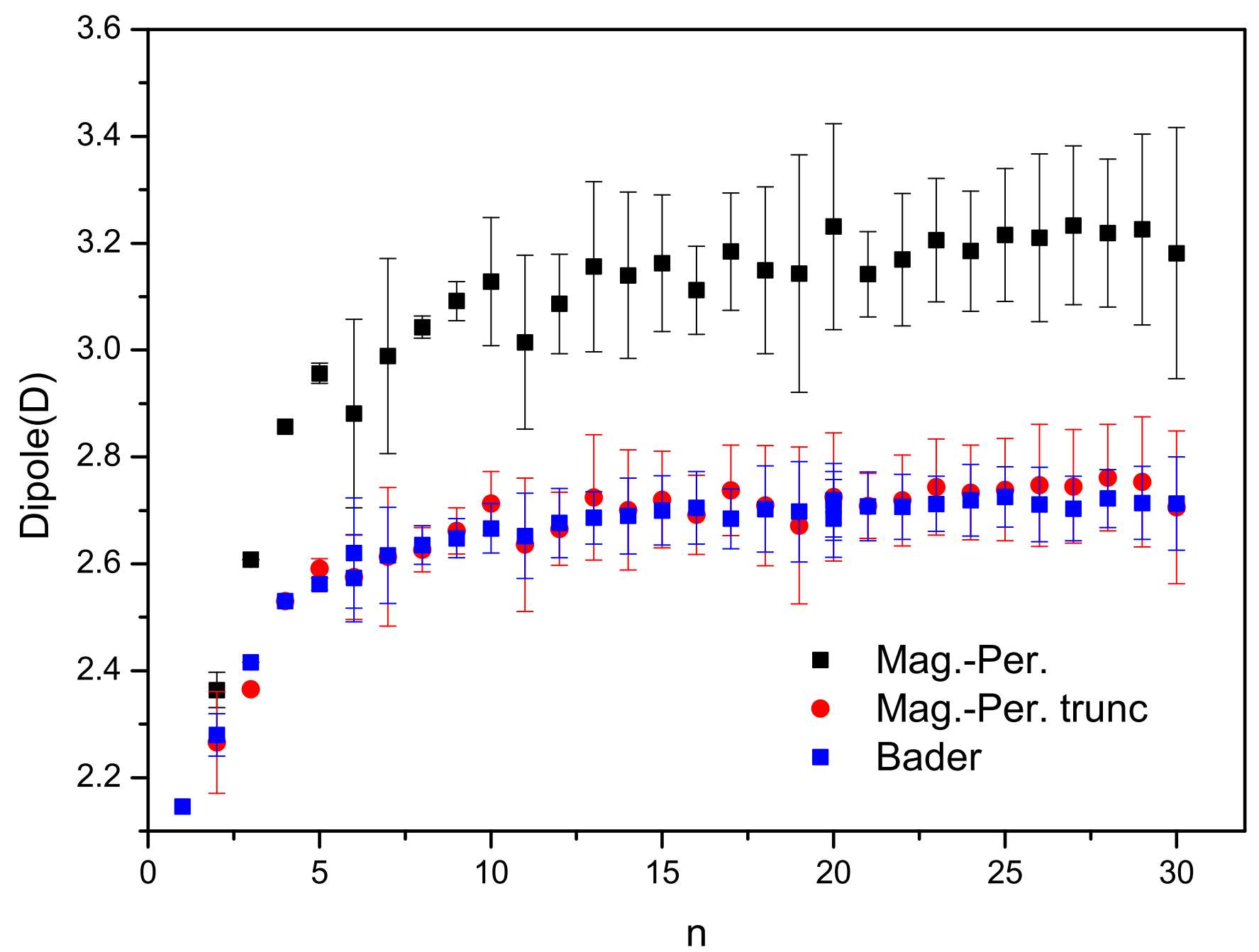

Figure 2 


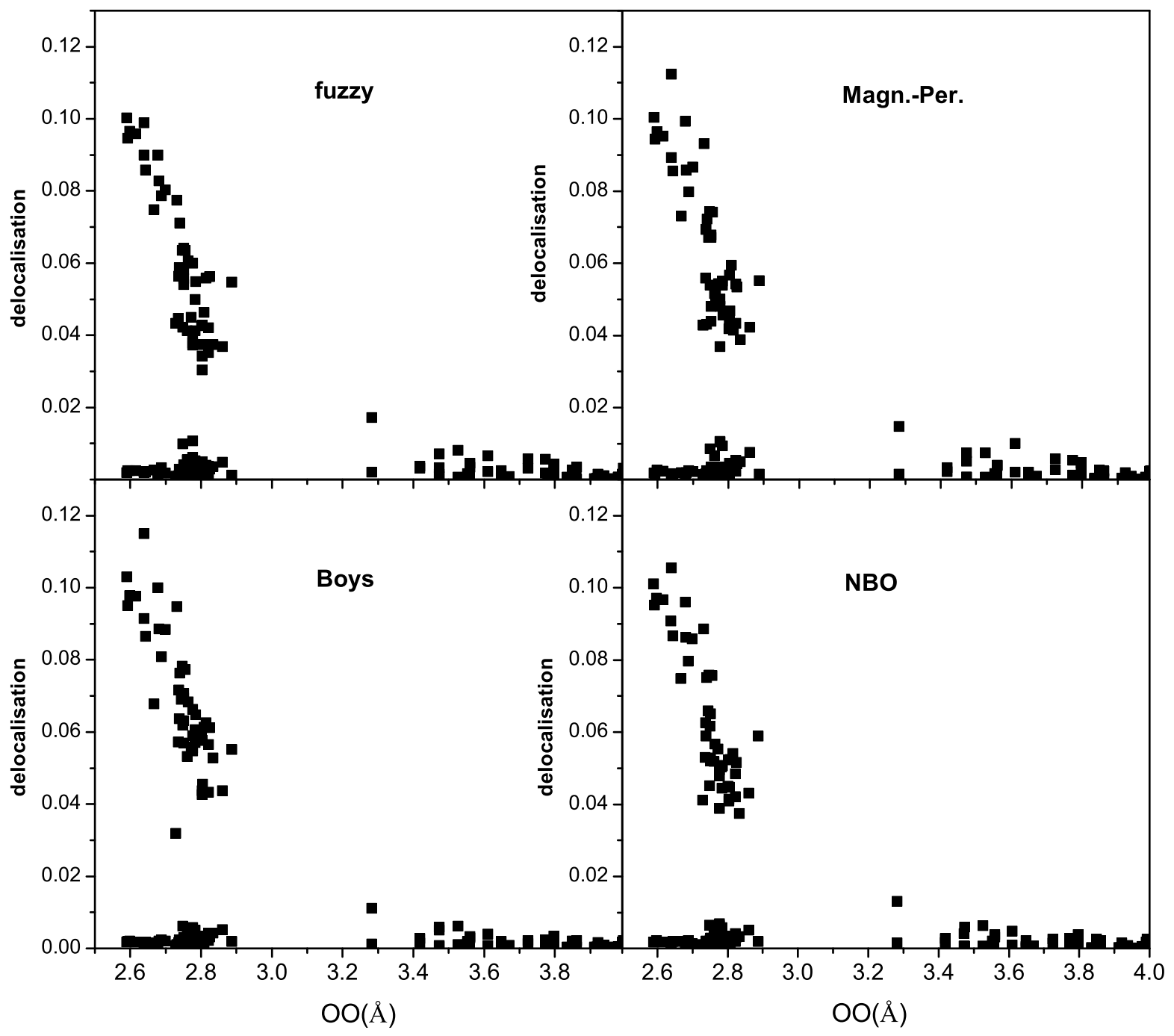

Figure 3 


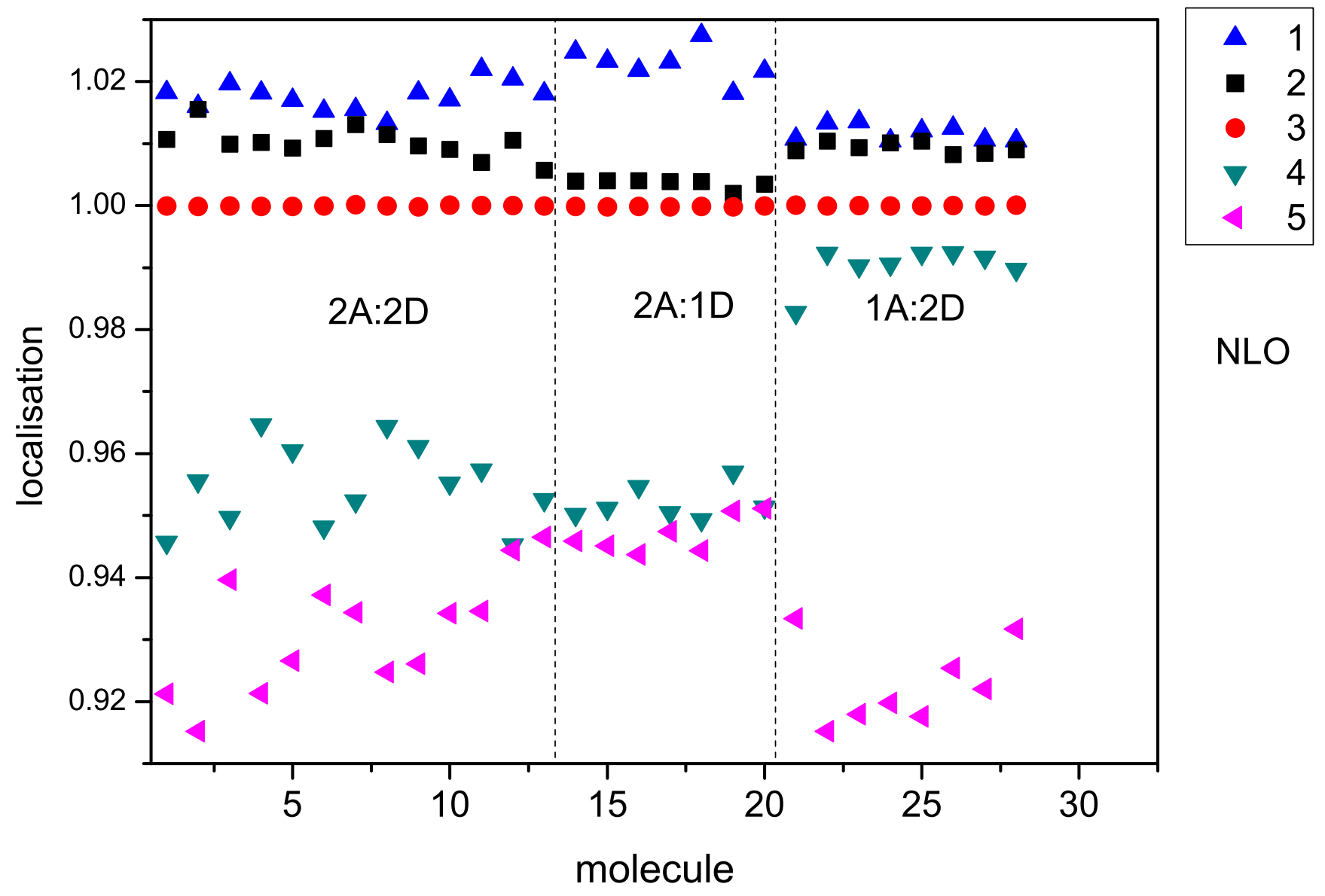

Figure 4 


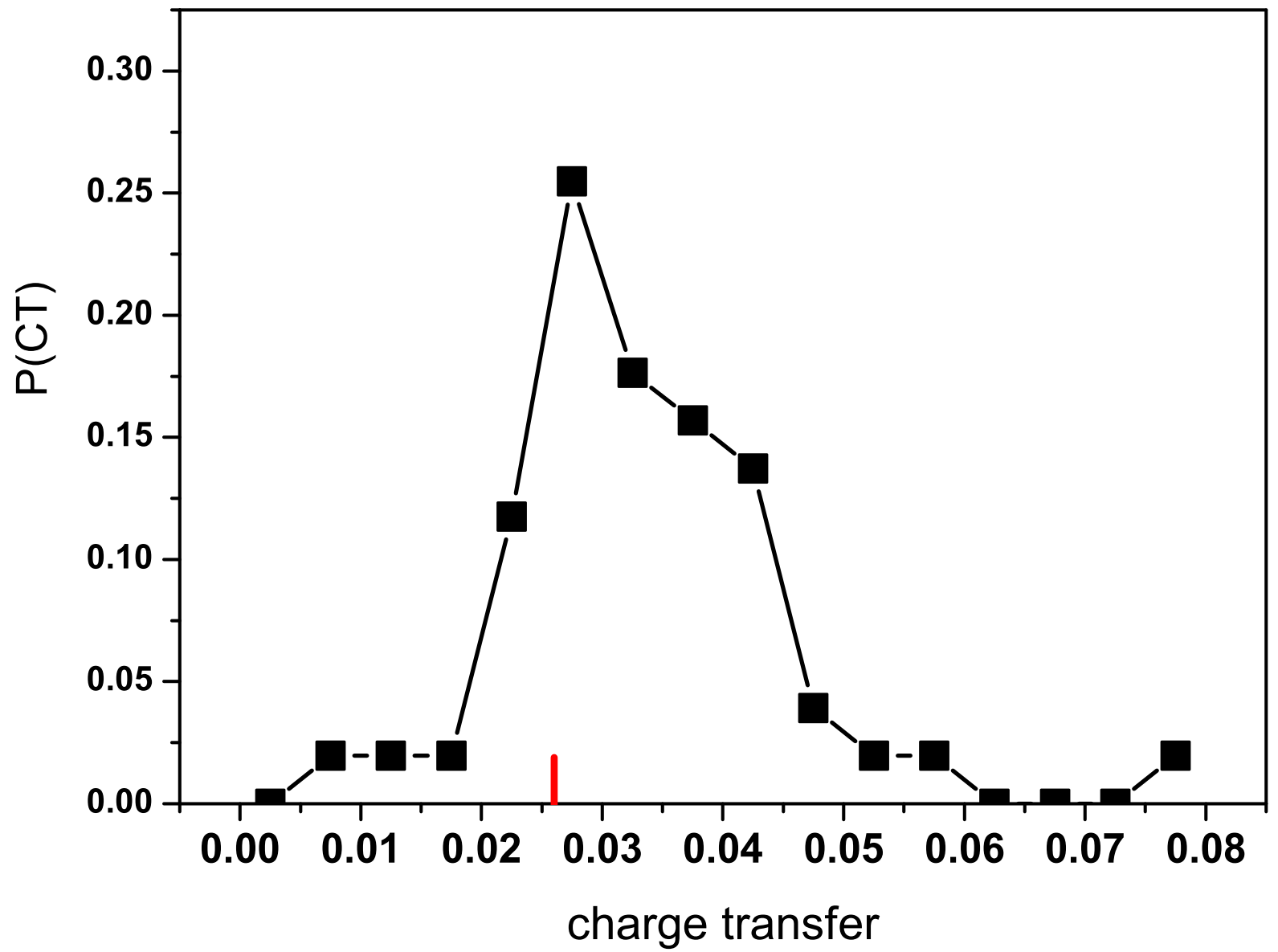

Figure 5 


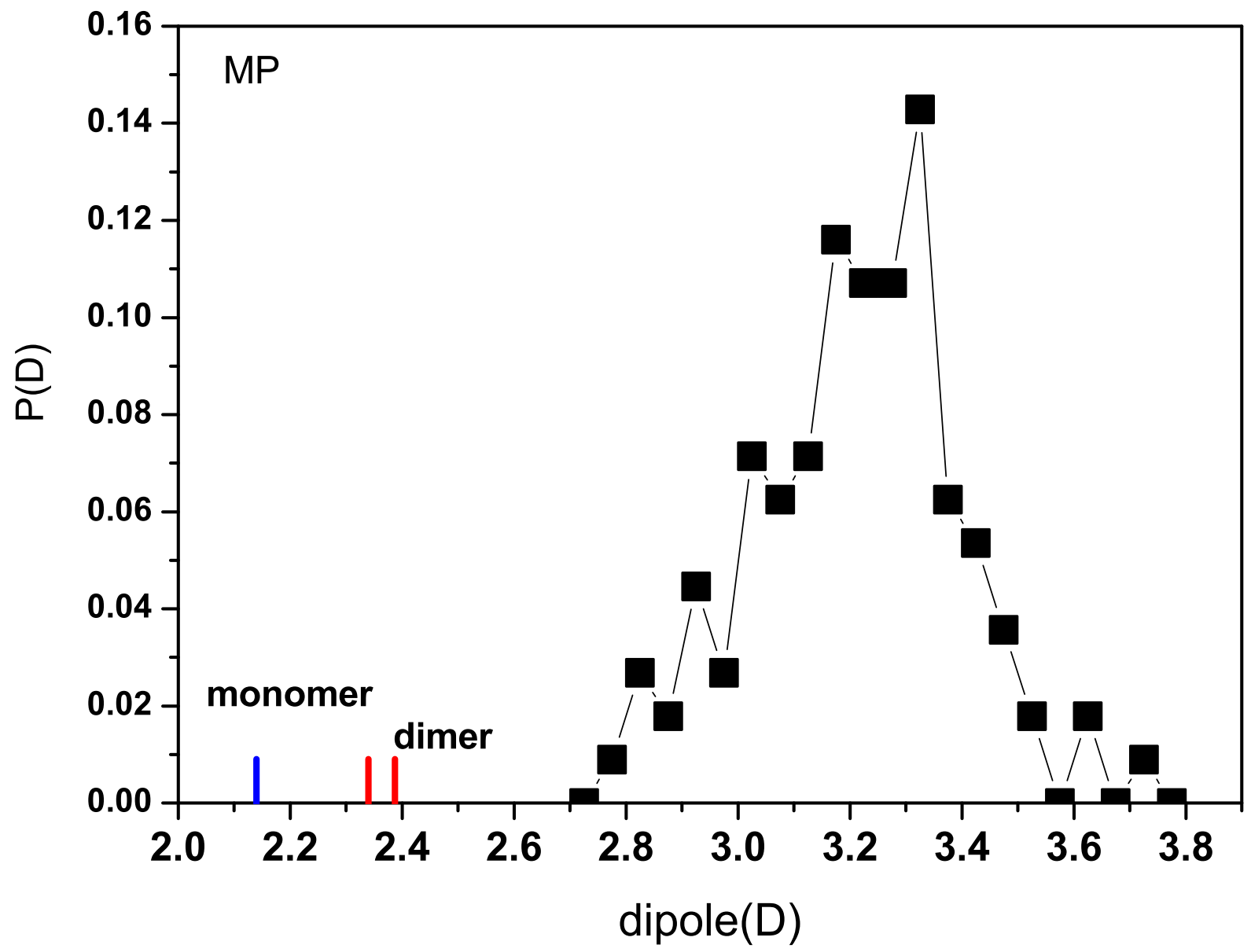

Figure 6 


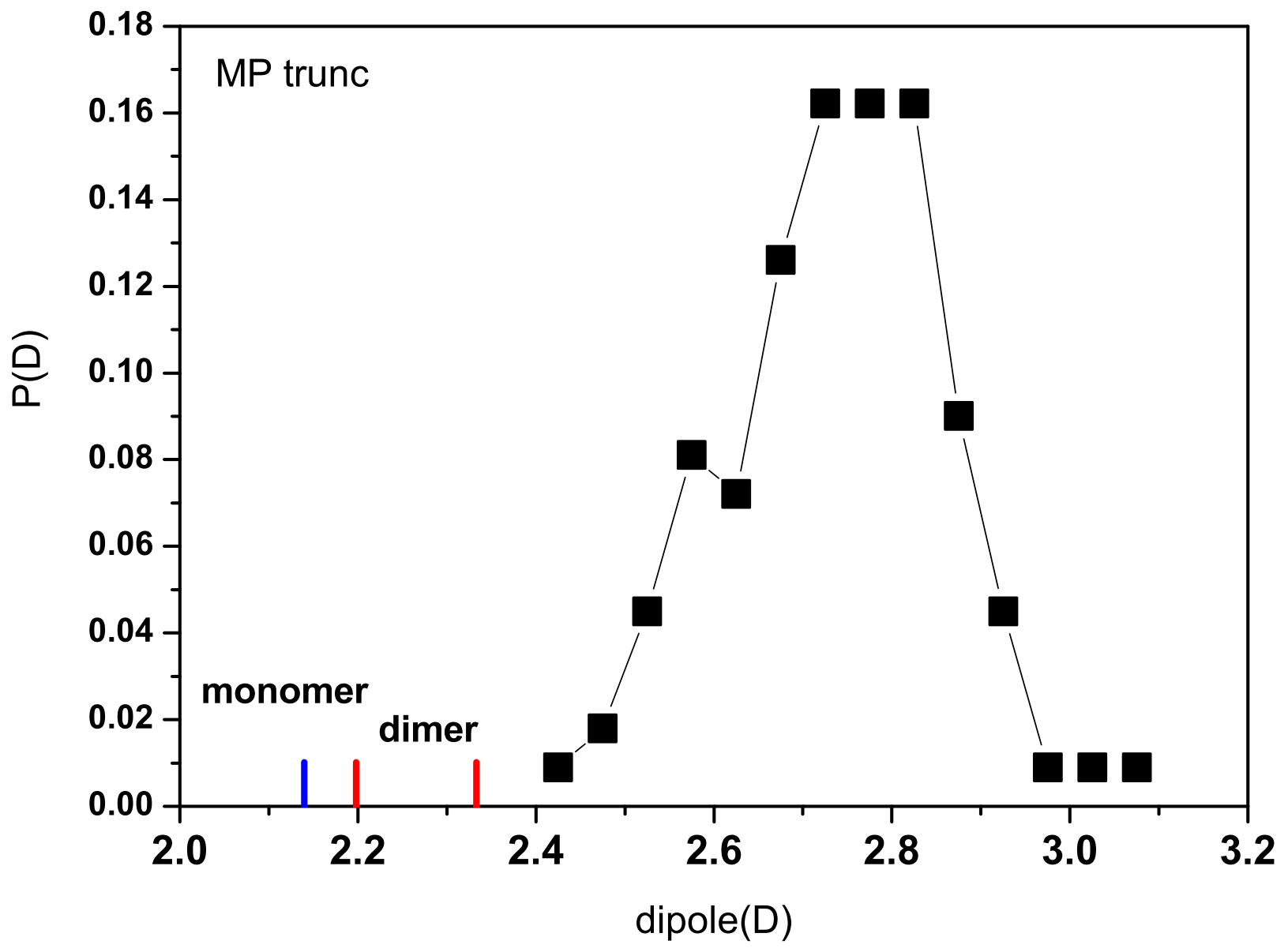

Figure 7 


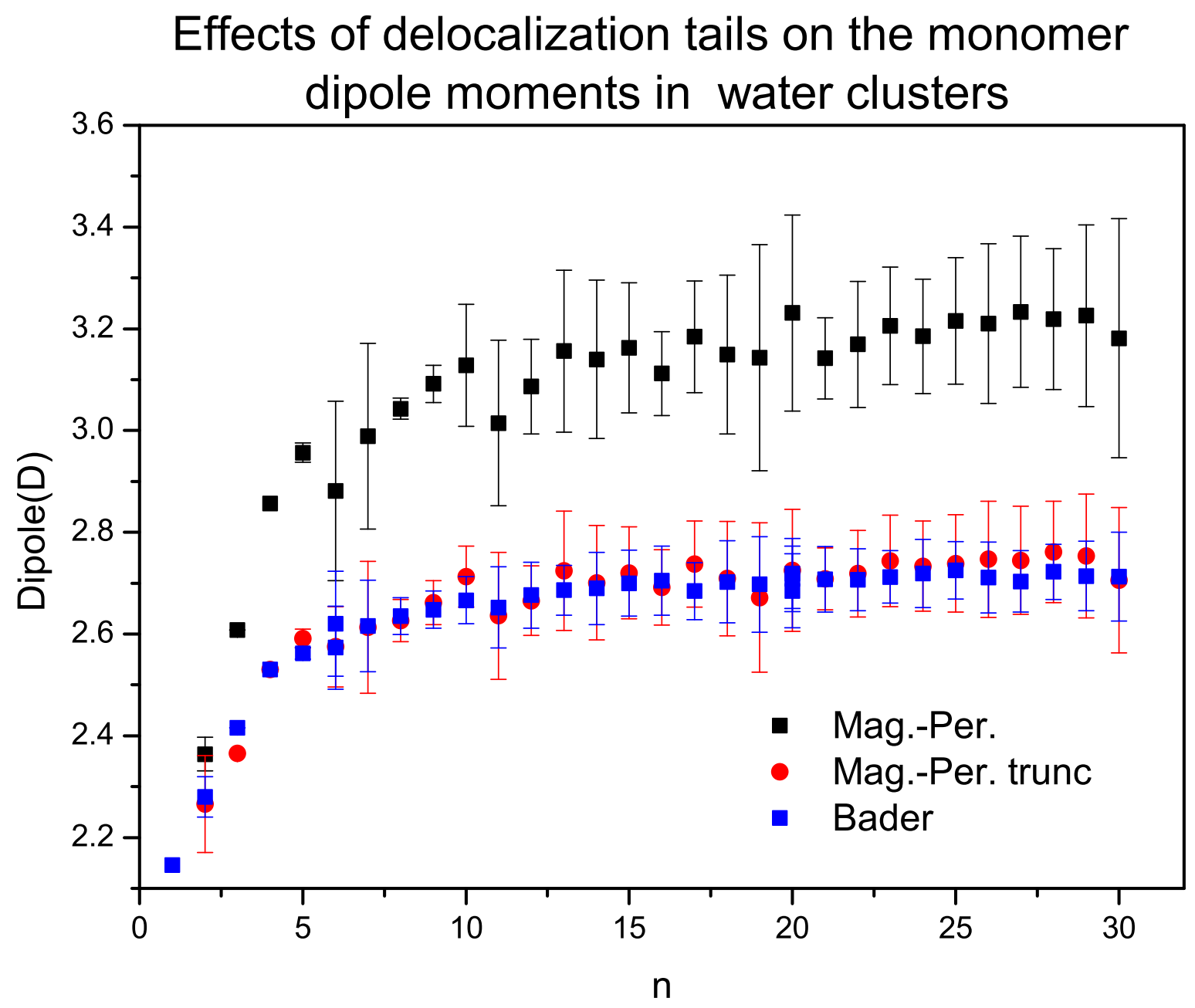

TOC graphics 